\title{
Surgery of congenital breast asymmetry-which objective parameter influences the subjective satisfaction with long-term results
}

\author{
Vivien Noisser ${ }^{1}$ D $\cdot$ Andreas Eigenberger ${ }^{1,2} \cdot$ Maximilian Weiherer $^{3} \cdot$ Stephan Seitz $^{4} \cdot$ Lukas Prantl $^{1}$. \\ Vanessa Brébant ${ }^{1}$ iD
}

Received: 11 June 2021 / Accepted: 20 August 2021 / Published online: 3 September 2021

(c) The Author(s) 2021

\begin{abstract}
Purpose Congenital breast asymmetry is a serious gynecological malformation for affected patients. The condition hits young women in puberty and is associated with socio-esthetic handicap, depression, and psychosexual problems. Surgical treatment is usually early in the patient's lifetime, so a long-term sustainable solution is important. Although postoperative outcome has been evaluated in several studies before, this study is the first to analyze which objective parameters have the greatest influence on subjective satisfaction with long-term results.

Methods Thirty-four patients diagnosed with congenital breast asymmetry that underwent either lipofilling or implant therapy between the years of 2008 to 2019 were examined. On average, our collective comprised patients seven years after surgery. Data were mainly gathered through manual measurements, patient-reported outcome measures (Breast $\mathrm{Q}^{\mathrm{TM}}$ ), and breast volumetry based on 3D scans (Vectra ${ }^{\circledR} \mathrm{H} 2$, Canfield Scientific).

Results Among all analyzed parameters, only areolar diameter correlated significantly negatively with the subjective outcome satisfaction of the patient. Regarding the subjective assessment of postoperative satisfaction with similarity of the breasts, again the mean areolar diameter, but also the difference in areolar diameter and breast volume between the right and left breasts correlated significantly negatively.

Conclusion Areolar diameter was revealed as being a significant factor influencing subjective long-term satisfaction in breast asymmetry patients. Moreover, 3D volumetry proves to be an effective tool to substantiate subjective patient assessments. Our findings may lead to further improvements to surgical planning and will be expanded in further studies.
\end{abstract}

Keywords Congenital breast asymmetry $\cdot$ Poland syndrome $\cdot$ Lipofilling $\cdot$ Silicone implant $\cdot 3 \mathrm{D}$ volumetry $\cdot$ Breast $\mathrm{Q}^{\mathrm{TM}}$

\section{Introduction}

Vanessa Brébant

vanessa.brebant@klinik.uni-regensburg.de

1 University Centre for Plastic, Aesthetic, Hand and Reconstructive Surgery, University Hospital Regensburg, Franz-Josef-Strauß-Allee 11, 93053 Regensburg, Germany

2 Faculty of Mechanical Engineering, Ostbayerische Technische Hochschule Regensburg (OTH Regensburg), Regensburg, Germany

3 Regensburg Medical Image Computing (ReMIC), Ostbayerische Technische Hochschule Regensburg (OTH Regensburg), Regensburg, Germany

4 Department of Obstetrics and Gynecology, Caritas Hospital St. Josef, University of Regensburg, Regensburg, Germany
The female breast plays an essential role in defining the human silhouette. Not the size of the breast is crucial for esthetics, but the overall appearance [1], which is largely determined by symmetry [2]. Congenital breast asymmetry thus represents a serious malformation to patients with the condition, with young women in puberty particularly affected. In this phase of life, humans are usually vulnerable, self-critical of their bodies, and anxious of how they compare with their peer group [3]. The breast is considered as a symbol of femininity, attractiveness, sexuality, and fertility. Any deformation of the breast is associated with a reduction in or loss of these values and is often viewed as a socio-esthetic handicap. Especially psychological symptoms such as depression and psychosexual problems burden the patients [4-7]. However, it is important to note that breast 
asymmetry to some degree is natural, and small differences in volume between the right and left breasts may be detected in nearly all women [8-10]. In contrast, obviously recognizable breast asymmetry should be classified as a deformity [8]. The specificity of the patient group lies in the phenotyping during puberty $[11,12]$. As a direct result of the described impairments, women with breast asymmetry feel a strong urge to undergo plastic surgery [3]. Most experts recommend early correction to avoid both emotional and social problems, such as stigmatization $[11,13]$. In several studies, affected women demonstrated a significant improvement in their subjective psychological stability after surgical correction. In addition, even in undressed situations, an increase in self-esteem has been reported [3, 14]. In general, treatment takes place relatively early on in the patient's life, so a longterm and sustainable solution is of particular importance to the patient [12].

Asymmetrical breasts still present a major challenge in plastic surgery [4]. Since the patient and the surgeon may evaluate a favorable outcome differently $[15,16]$, this study analyzes which objective factors influence subjective long-term satisfaction in patients with congenital breast asymmetry.

\section{Materials and methods}

\section{Recruitment and participants}

Prior to participant recruitment, the ethics committee of Regensburg University approved the study (approval number: 20-1654-101). Patients diagnosed with breast asymmetry and who underwent correction surgery involving either lipofilling or silicone implant at our institution (University Center for Plastic, Aesthetic, Hand and Reconstructive Surgery, Regensburg) between the years of 2008 and 2019 were included in the study. Women who were minors at the time of the retrospective data collection, had epilepsy, had undergone mastectomy, or patients with acquired breast asymmetry were excluded from the study. The data were collected between March and July 2020. A compact overview of our patient collective is portrayed in Table 1.
Half of the patients were treated with lipofilling and the other half with silicone implant augmentation. Our cohort included five women diagnosed with Poland syndrome, fifteen patients with tuberous breast deformity and breast asymmetry, thirteen women with Amazon's syndrome, and one patient with chest deformity. At the time of surgery, ten out of 34 patients were minors. At the time of data collection (on average seven years later), all 34 patients were adults.

\section{Study design}

Prior to participating, all patients provided their informed consent.

Data for the following objective and subjective parameters were collected during clinical examination. The cup size (A/B/C/D/E) was determined by medical assessment. The scar quality was evaluated on a scale from 1 to 3 (hardly/ moderate/highly visible scars). As body measurements can be an important predictor of female attractiveness [17], the patient was measured manually with a classic tape measure along the skin surface. The following measurements were recorded: sternal notch to nipple $(\mathrm{SN}-\mathrm{N})$, inframammary fold to nipple (IMF-N), upper breast pole to nipple (UBP-N), xiphoid to nipple (Xi-N), lateral breast pole to nipple (LB-N), inframammary fold length (IMF-length) and areolar diameter (AD). Figure 1 depicts the recorded measurements, which were taken as the shortest distance along the skin surface.

\section{Three-dimensional volumetry}

Three-dimensional breast volumetry was performed with the portable Vectra ${ }^{\circledR} \mathrm{H} 2$ (Canfield Scientific, USA), which is frequently used in the literature [18-26]. The 3D model can be analyzed in terms of breast volume and various breast dimensions by using the Breast sculptor ${ }^{\circledR}$ software package. Based on a modified protocol, O'Connell et al. [20] conducted a validation study using the Vectra ${ }^{\circledR}$ XT, in which the anterior axillary line was selected as the lateral breast boundary, see Fig. 2. We integrated these findings, in order to achieve the best possible reproducibility in our study:
Table 1 Description of our patient collective

\begin{tabular}{lll}
\hline$n=34$ & Mean $( \pm$ SD) & Range \\
\hline Age (at time of data collection) [in years] & $30( \pm 5.8)$ & $21-45$ \\
Age (at time of first breast surgery) [in years] & $21( \pm 5.6)$ & $16-42$ \\
BMI (at time of last breast surgery) [in kg/m $\left.{ }^{2}\right]$ & $23.6( \pm 4.1)$ & $18-38$ \\
Length of postoperative period [in years] & $7( \pm 3.3)$ & $0.9-12$ \\
Cup size & $\mathrm{C}$ & A-E \\
Scar quality [given a scale from 1 (best) to 3 (worst)] & $1.4( \pm 0.6)$ & $1-3$ \\
Number of surgery sessions & $2( \pm 1.2)$ & $1-5$ \\
\hline
\end{tabular}




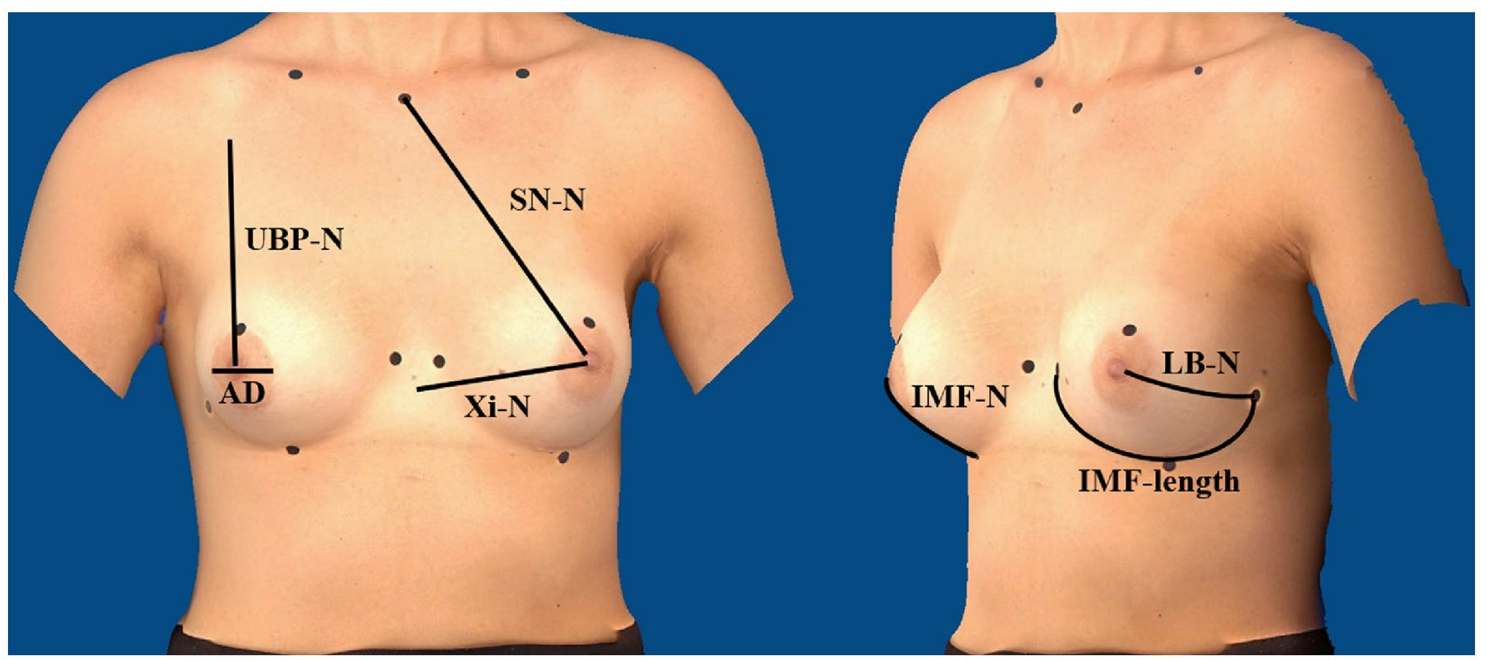

Fig. 1 Detailed illustration of measurements (picture created with Canfield Vectra ${ }^{\circledR} \mathrm{H} 2$ and edited), patient with implant therapy

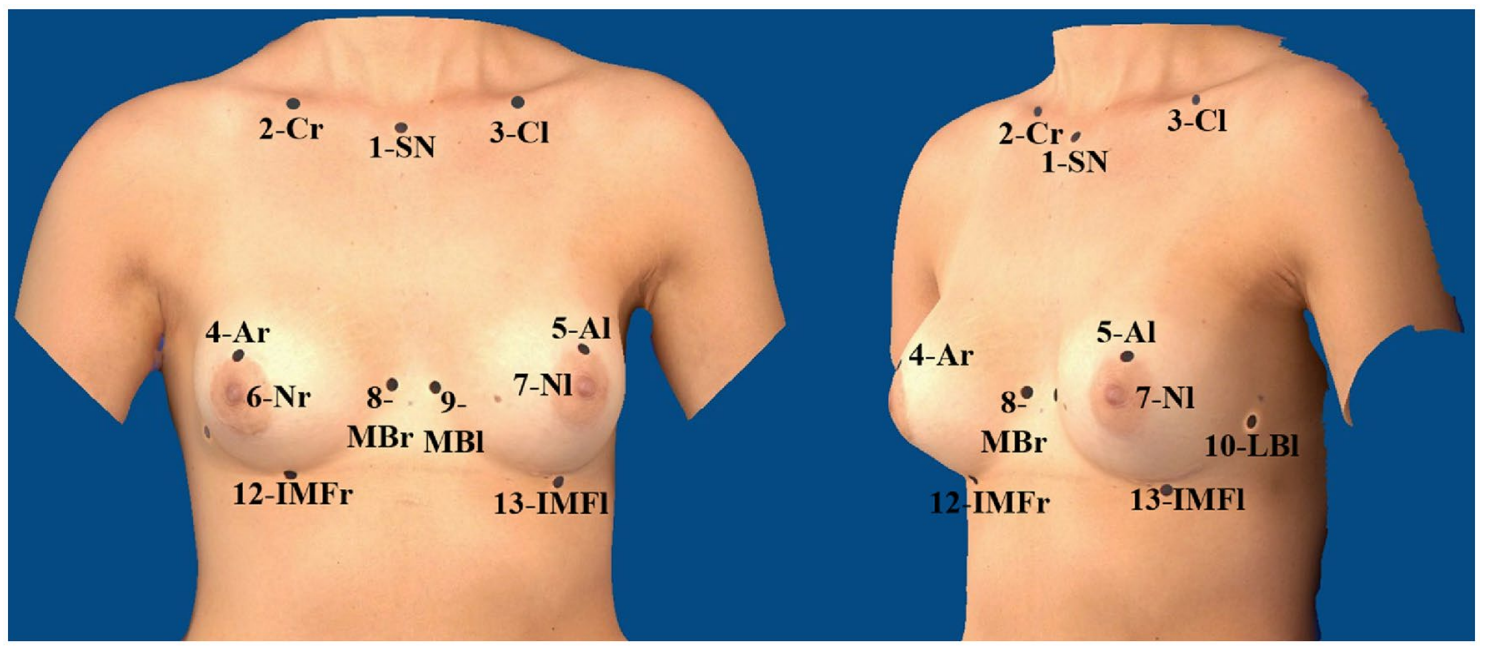

Fig. 2 Reference points for Canfield Vectra ${ }^{\circledR} \mathrm{H} 2$ (picture created with Canfield Vectra ${ }^{\circledR} \mathrm{H} 2$ and edited), patient with implant therapy

(1) Sternal notch $(\mathrm{SN}),(2)$ center of the right clavicle (Cr), (3) center of the left clavicle (Cl), (4) most cranial point of the right areola (Ar), (5) most cranial point of the left areola (Al), (6) right nipple ( $\mathrm{Nr})$, (7) left nipple ( $\mathrm{Nl})$, (8) end of right medial inframammary fold (MBr), (9) end of left medial inframammary fold (MBl), (10) end of right lateral inframammary fold ( $\mathrm{LBr}),(11)$ end of left lateral inframammary fold (LBl), (12) most caudal point of the right inframammary fold (IMFr), (13) most caudal point of the left inframammary fold (IMFl).

Points (8) and (9) are seen as the medial border of the breast. They are more precisely defined as the point of the inframammary fold with the shortest distance to the anterior median line. The lateral border of the breast is marked by points (10) and (11). As the appearance of the breast varies greatly from woman to woman [27], depending on the shape of the breast, the inframammary fold can be highly variable and might end diffusely [28], which makes reproduction of the present reference point inaccurate. The high inaccuracy of reference points was also criticized by O'Connell et al. [20]. They defined the lateral breast boundary as the anterior axillary line [20]. To use a reproducible reference point and to achieve uniformity with manual volumetry, we decided to follow O'Connell et al. [20] and defined the point of the lateral breast boundary as the intersection of the anterior axillary line with a line through the nipple.

All images were taken at our institute, processed and overlayed, and analyzed using Vectra ${ }^{\circledR}$ and Breast Sculptur ${ }^{\circledR}$. The camera equipment includes a special positioning mat, precisely specifying the position of the patient and 
photographer for each of the three images. The $45^{\circ}$ angle of the arms was checked with a goniometer. In addition, a telescopic stick was used to assist the patient with maintaining the requested position of the arms fixed. The first and third images capture the patient at a $45^{\circ}$ angle, from the right and left, respectively. The second image is taken frontally. The Vectra ${ }^{\circledR}$ software then assembles a 3D model from the images. In order to perform calculations on the resulting 3D model, 13 reference points are required. As the automatic detection of the reference points by the Vectra ${ }^{\circledR}$ software did not work as well as expected, the reference points were marked on the patient before the images were taken and then set accordingly in the software. This seems to be a common problem, which is well described by other researchers [19]. Following Eder et al. [29], we used the positive effects of pre-marking the reference points [29].

\section{Patient-related-outcome measures: Breast $Q^{\text {tm }}$ questionnaire}

All participants completed the Breast $\mathrm{Q}^{\mathrm{TM}}$ questionnaire (Breast Q Version 2.0 ${ }^{\odot}$, Augmentation Modules Pre- and Postoperative Scales, German (DE) Version, The University of British Columbia, licensed for non-profit users by Memorial Sloan Kettering Cancer Center and translated by Mapi Research Trust, 2008). The Breast $\mathrm{Q}^{\mathrm{TM}}$ is a standardized, patient-reported outcome measure (PROM) on subjective quality of life, developed by Pusic et. al. [30]. Over five years, Breast $Q^{\mathrm{TM}}$ was validated with the help of about 3000 women [31]. It is thus considered a clinically relevant, standardized outcome evaluation instrument that meets psychometric criteria and captures the patient's self-assessment according to a state-of-the-art system [31]. Recently, Breast $\mathrm{Q}^{\mathrm{TM}}$ was also used by other researchers in similar studies $[32,33]$.

This study implemented the module probing the patient's satisfaction after breast surgery. It evaluates the satisfaction with the breast surgery outcome by means of eight questions with three possible answers for each: $1=$ disagree; $2=$ somewhat agree; $3=$ completely agree. Using the enclosed transformation score, the sum for each module may be interpreted directly as a value between 0 (worst value) and 100 (best value), effectively providing a percentage "score of satisfaction".

\section{Statistical analysis}

Statistical evaluation was carried out with SPSS ${ }^{\circledR}$ Statistics Version 25.0.0. from IBM ${ }^{\circledR}$. Using Spearman's correlation, all meaningful parameters were examined for any correlation with patient subjective outcome satisfaction. Spearman's correlation was chosen because either at least one parameter was scaled ordinally, or at least one outlier was found. A significance level of 0.05 was considered.

\section{Results}

\section{Correlations of objective parameters with long-term subjective satisfaction}

The results of the Breast- $\mathrm{Q}^{\mathrm{TM}}$ questionnaire are portrayed in Table 2.

As seen, none of the objective parameters investigated correlates significantly with long-term outcome satisfaction.

The results for the manually measured anthropometric distances are depicted in Table 3.

Specifically, we tested whether a distance (mean value (MV) or difference $(\Delta)$ between the right and left sides) had any influence on subjective outcome satisfaction or the perception of breast similarity in the long-term observation. The latter is a single question concerning the patient's subjective satisfaction with the similarity of her breasts (on a scale from 1 (worst) to 4 (best)).

Regarding the mean values of the areolar diameter (MV AD), there was a significant correlation between both the general satisfaction with the result $\left(r_{s}=-0.405\right.$; $p$ value $=0.017)$ as well as the subjective assessment of similarity between the right and left breasts $\left(\mathrm{r}_{\mathrm{s}}=-0.355\right.$; $p$ value $=0.039)$. No other mean values of the body measurements ( SN-N, IMF-N, UBP-N, Xi-N, LB-N, IMFLength) had any significant influence on the subjective satisfaction result or the self-perception of similarity between both breasts. The larger the mean value of the areolar diameter, the more dissatisfied the patients were

Table 2 Spearman's correlation of outcome satisfaction with surveyed parameters

\begin{tabular}{|c|c|c|}
\hline \multirow[t]{2}{*}{$n=34$} & \multicolumn{2}{|c|}{$\begin{array}{l}\text { Breast } Q^{\mathrm{TM}} \\
\text { satisfaction with } \\
\text { outcome }\end{array}$} \\
\hline & $\mathrm{r}_{\mathrm{s}}$ & $p$ value \\
\hline Age (at time of data collection) [in years] & 0.260 & 0.137 \\
\hline Age (at time of first breast surgery) [in years] & 0.000 & 0.999 \\
\hline BMI (at time of last breast surgery) $\left[\mathrm{in} \mathrm{kg} / \mathrm{m}^{2}\right]$ & -0.179 & 0.310 \\
\hline Length of postoperative period [in years] & -0.80 & 0.653 \\
\hline Cup size & -0.136 & 0.443 \\
\hline $\begin{array}{l}\text { Scar quality (on a scale from } 1 \text { (best) to } 3 \\
\text { (worst)) }\end{array}$ & -0.113 & 0.523 \\
\hline Number of surgery sessions & 0.234 & 0.183 \\
\hline
\end{tabular}


Table 3 Results of Spearman's correlation for body measurement parameters (mean and $\Delta$ between the right and left breasts), objective volumetry, and symmetry index with outcome satisfaction or satisfaction with similarity of breasts

\begin{tabular}{|c|c|c|c|c|}
\hline \multirow[t]{2}{*}{$n=34$} & \multicolumn{2}{|c|}{$\begin{array}{l}\text { Breast } Q^{\mathrm{TM}} \text { satisfac- } \\
\text { tion with similarity of } \\
\text { breasts }\end{array}$} & \multicolumn{2}{|c|}{$\begin{array}{l}\text { Breast } Q^{\mathrm{TM}} \\
\text { satisfaction with } \\
\text { outcome }\end{array}$} \\
\hline & $\mathrm{r}_{\mathrm{s}}$ & $p$ value & $r_{s}$ & $p$ value \\
\hline MV SN-N & -0.159 & 0.370 & -0.300 & 0.084 \\
\hline MV IMF-N & 0.071 & 0.689 & -0.119 & 0.504 \\
\hline MV UBP-N & -0.194 & 0.272 & -0.039 & 0.826 \\
\hline MV Xi-N & -0.115 & 0.519 & -0.186 & 0.293 \\
\hline MV LB-N & -0.305 & 0.079 & -0.153 & 0.387 \\
\hline MV AD & $-0.355^{*}$ & 0.039 & $-0.405^{*}$ & 0.017 \\
\hline MV IMF-length & -0.168 & 0.343 & -0.314 & 0.071 \\
\hline$\Delta \mathrm{SN}-\mathrm{N}$ & -0.269 & 0.123 & 0.098 & 0.580 \\
\hline$\Delta \mathrm{IMF}-\mathrm{N}$ & -0.137 & 0.441 & -0.043 & 0.811 \\
\hline$\Delta \mathrm{UBP}-\mathrm{N}$ & -0.187 & 0.290 & -0.054 & 0.761 \\
\hline$\Delta \mathrm{Xi}-\mathrm{N}$ & -0.287 & 0.100 & -0.283 & 0.105 \\
\hline$\Delta \mathrm{LB}-\mathrm{N}$ & 0.002 & 0.989 & -0.176 & 0.320 \\
\hline$\Delta \mathrm{AD}$ & $-0.381 *$ & 0.026 & -0.242 & 0.168 \\
\hline$\Delta$ IMF-length & 0.076 & 0.669 & -0.138 & 0.435 \\
\hline$\Delta 3 \mathrm{D}$ volume & $-0.389 *$ & 0.023 & -0.231 & 0.189 \\
\hline $\begin{array}{l}\text { Satisfaction with } \\
\text { similarity of } \\
\text { breasts }\end{array}$ & 1 & 0 & $0.597 * *$ & $<0.01$ \\
\hline
\end{tabular}

$\mathrm{r}_{\mathrm{s}}=$ correlation coefficient

*Significant $(p<0.05)$

$* *$ Highly significant $(p<0.01)$

with the long-term result and the more dissimilar they felt their two breasts to be.

Regarding the difference between the right and left breasts $(\Delta)$, we only found significant correlation for the $\Delta$ in the areolar diameter $(\Delta \mathrm{AD})$. This correlated negatively with the patient's subjective satisfaction with the similarity of her breasts ( $p$ value $=0.026 ; \mathrm{r}_{\mathrm{s}}=-0.381$ ). Clearly, the smaller the difference between the areolar diameters of the right and left breast, the more satisfied the patient was with the similarity of her breasts. In addition, the question considering satisfaction with similarity of the breasts correlated strongly with the overall satisfaction with outcome. The $p$ value was less than 0.01 and the correlation coefficient 0.597 . We conclude that the satisfaction with the similarity of the breasts has a huge impact on the satisfaction with the overall, long-term result. None of the other calculated values for $\Delta$ of the body measurements (SN-N, IMF-N, UBP-N, Xi-N, LB-N, IMF-length) had any significant influence on the resulting satisfaction or the satisfaction with the similarity of the breasts.
Finally, the smaller the volume difference between the right and left breasts, the more satisfied the patient was with the similarity of her breasts ( $p$ value $=0.023 ; \mathrm{r}_{\mathrm{s}}=-0.389$ ).

\section{Discussion}

\section{Discussion of methods}

In comparison with other authors in this field who tend to assess patient satisfaction between a few months and a maximum of one year postoperatively [14, 24, 34], we examined the patients in our study on average seven years postoperatively. This made it possible to attach comparatively greater importance to the long-term outcome. However, the disadvantage of such long-term observation lies in generation of the study collective. Although our study collective of 34 patients is not unusual compared to similar studies of congenital breast asymmetry (Kuzbari et al. [35] $n=30$; Neto et al. [14] $n=35$; Eder et al. [29] $n=28$ ), the small collective should be viewed critically, as it allows only limited generalizations. Therefore, a multicenter approach may prove advantageous in follow-up work in order to generate a larger collective.

We assessed long-term outcome satisfaction using the validated Breast $\mathrm{Q}^{\mathrm{TM}}$ questionnaire, which was developed specifically for breast augmentation [30] and corresponds to the current state of research [36]. Especially in earlier studies, some authors worked with a simple satisfaction scale $[10,35,37,38]$ or used measurement tools that did not specifically refer to the breast [14]. In recent years, however, the breast-specific Breast $Q^{\mathrm{TM}}$ has increasingly replaced these and is used frequently in research [24, 32-34]. Critically, however, it should be noted that long-term outcome satisfaction as assessed by the Breast $\mathrm{Q}^{\mathrm{TM}}$ is a very complex construct [39] and may be influenced by many other individual factors besides those assessed in this study. For instance, many people consider plastic surgery as a cure for their personal and relationship problems [40]. This may have created a bias in long-term outcome satisfaction. Another drawback of the Breast $Q^{\mathrm{TM}}$ is that the sensitivity of the breast after surgery is not included in the questionnaire. However, this appeared to be particularly relevant in our study. Some patients reported numbness or hypersensitivity as one of their biggest postoperative problems. We, therefore, recommend including breast sensitivity in follow-up work.

By employing a state-of-the-art 3D scanning technique to assess objective outcome, we base the methodology of our study on the latest research. In the current literature, stateof-the-art 3D volumetry has been evaluated as both objective and effective [41, 42]. Regarding volumetry with Vectra ${ }^{\circledR} \mathrm{H} 2$ from Canfield, however, it should also be noted that there are some limitations. The camera is well suited to creating 
a 3D model of small to medium-sized breasts and to analyzing their volumes. However, for larger and ptotic breasts, the most caudal point of the inframammary fold, an essential reference point for Canfield's Vectra ${ }^{\circledR} \mathrm{H} 2$ (see methods), is not visible and obscured by the breast itself. This limited proper volumetry in this study, as was the case for Koban et al. in 2018 [41]. In summary, the methodology of our study is characterized not only by its proximity to the current state of research, but also by the comprehensive survey of subjective and objective factors and the long-term observation of the rare diagnosis of congenital breast asymmetry.

\section{Discussion of results}

In our study, volumetry with the Vectra ${ }^{\circledR} \mathrm{H} 2$ by Canfield proved to be an effective tool to quantify a patient's subjective satisfaction in a side-by-side comparison of the right and left breasts (Figs. 3 and 4). We were thus able to confirm the results of Ji et al. [42] proving the 3D-scan technique to be an objective and effective tool for analyzing and documenting breast morphology (Figs. 3 and4). In addition, we were also able to support the results of Eder et al. [29] regarding the increasing relevance of 3D technology in the comparison of breast augmentation therapies with this study.

We found the mean value of the areolar diameter to be remarkable, as it correlated significantly with the patient's subjective assessment of similarity between their breasts as well as with the long-term outcome. Furthermore, our study confirmed the findings of Osinga et al. [38] that neither scar appearance nor breast size had any significant influence on the overall outcome. In everyday clinical practice, the $\mathrm{SN}-\mathrm{N}$ distance is considered the most important parameter. However, we did not reveal that a smaller $\Delta \mathrm{SN}-\mathrm{N}$ had any positive effect on the subjective symmetry score for the patient. Our results differ from Osinga et al. on this aspect [38]. The mean areolar diameter (MW AD) was the only body measurement in our work that had a significant influence on the long-term outcome. With this finding, our study supports the results of Pietruski et al. [43], who recently demonstrated in their eye-tracking study that the nipple-areola complex is one of two key focus areas of the female breast.

\section{Conclusion}

The strength of our study lies in its employment of a stateof-the-art 3D-scanning technique with its accuracy of data collection and reliable registration compared to other methods. The combination of subjective and objective criteria in the evaluation of the outcome underlines the comprehensive approach of our study. The long-term follow-up design of our study is unique in this specific field of congenital breast deformity. It is a real advantage considering the young age of the patients at the time of surgery. Areolar diameter was
Fig. 3 Patient with congenital breast asymmetry (tubular deformity): the left image was taken prior to surgery in 2017. The right image was taken in 2020, therapy: implant
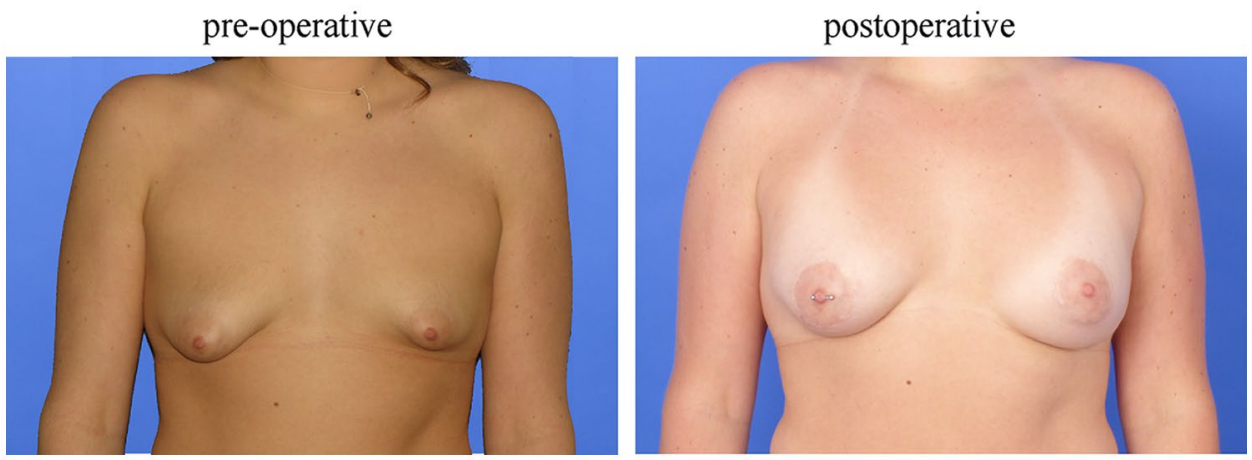

Fig. 4 Patient with breast asymmetry (Poland syndrome): the left image was taken prior to lipofilling therapy in two sessions (surgery: 2010 and 2013). The right image was taken in 2020
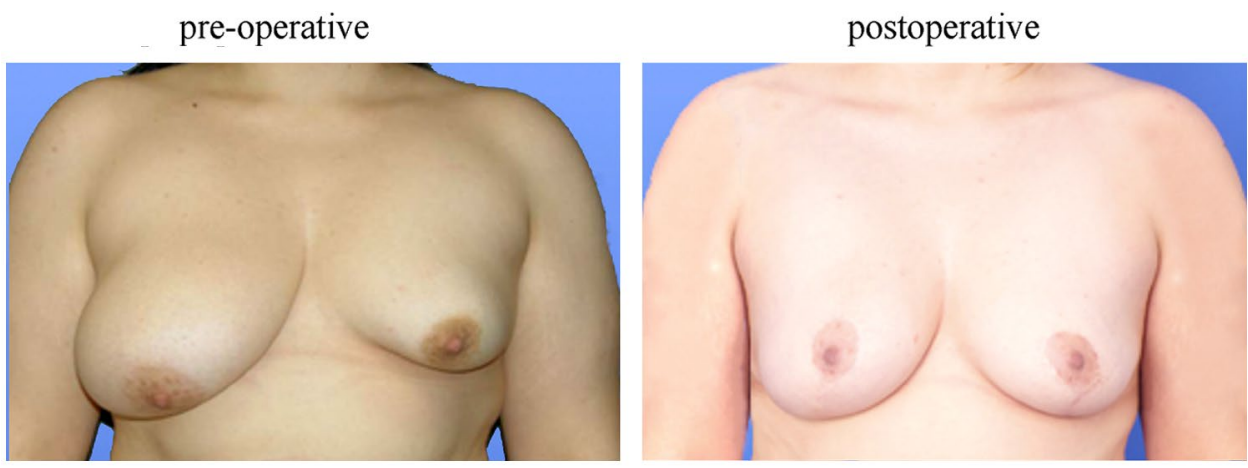
revealed as being a significant influencing factor in patientsubjective long-term satisfaction for breast asymmetry patients, which will be a focus of our future research and could lead to further improvement in surgical planning. One limitation of our study is the rather small collective, which is justified by the rare clinical picture and the long-term retrospective focus of the study. We thus plan to focus on multicentric studies, in order to generate a larger collective. Furthermore, a prospective design could help us to answer further questions considering breast sensitivity.

Our study takes us one step closer to the long-term goal of establishing robust instruments to evaluate the results of breast surgery and contribute to the quality assurance of breast surgery in congenital breast asymmetry.

Acknowledgements We thank Karolina Müller (Centre for Clinical Trials and Studies, Regensburg, Germany) for her consultation regarding statistical analysis. Furthermore, we would like to thank all our team members who supported this study and drove it forward.

Author contributions VN: conceptualization, data collection, data analysis and interpretation, original draft and writing of the article. AE: photo editing, critical revision of the manuscript, final approval. MW: critical revision of the manuscript, data preparation, final approval. SS: critical revision of the manuscript, final approval. LP: critical revision of the manuscript, final approval. VB: conceptualization, data collection, supervision, critical revision of the manuscript, final approval.

Funding Open Access funding enabled and organized by Projekt DEAL.

\section{Declarations}

Conflict of interest The authors declare that there are no conflicts of interest.

Ethical approval The institutional ethics committee of the University of Regensburg Faculty of Medicine approved this study (approval number: 20-1654-101). All procedures performed in studies involving human participants were in accordance with the ethics standards of the institutional research committee and with the 1964 Helsinki Declaration and its later amendments as well as comparable standards of ethics.

Informed consent Written informed consent was obtained from all individual participants included in the study.

Consent for publication The written consent to use patient photographic documentation and data for publication was obtained from all patients included in this study.

Open Access This article is licensed under a Creative Commons Attribution 4.0 International License, which permits use, sharing, adaptation, distribution and reproduction in any medium or format, as long as you give appropriate credit to the original author(s) and the source, provide a link to the Creative Commons licence, and indicate if changes were made. The images or other third party material in this article are included in the article's Creative Commons licence, unless indicated otherwise in a credit line to the material. If material is not included in the article's Creative Commons licence and your intended use is not permitted by statutory regulation or exceeds the permitted use, you will need to obtain permission directly from the copyright holder. To view a copy of this licence, visit http://creativecommons.org/licenses/by/4.0/.

\section{References}

1. Raposio E, Belgrano V, Santi P, Chiorri C (2016) Which is the ideal breast size? Some social clues for plastic surgeons. Ann Plast Surg 76:340-345. https://doi.org/10.1097/SAP.0000000000 000471

2. Kiene M, Hoch J (2004) Mammakarzinom und Lebensqualität Gewinn durch Rekonstruktion und Angleichung der Gegenseite? Handchir Mikrochir plast Chir 36:384-391. https://doi.org/10. 1055/s-2004-817951

3. NiMhurchadha S, Harcourt D, Diba R et al (2012) Looking 'the same': experiences of women who have had corrective surgery for breast asymmetry. J Health Psychol 18(4):488-496

4. Yesilada AK, Sevim KZ, Sirvan SS et al (2013) Our surgical approach to treatment of congenital, developmental, and acquired breast asymmetries: a review of 30 cases. Aesth Plast Surg 37:77-87. https://doi.org/10.1007/s00266-012-0041-9

5. Sarkar DK, Maji A, Saha S, Biswas JK (2011) Oncoplastic breast surgery - our experiences in the breast clinic, IPGME\&R, Kolkata. Indian J Surg Oncol 2:112-117. https://doi.org/10. 1007/s13193-011-0065-7

6. Rintala AE, Nordström REA (1989) Treatment of severe developmental asymmetry of the female breast. Scand J Plast Reconstr Surg 23:231-235. https://doi.org/10.3109/028443189090751 23

7. Nuzzi L, Cerrato F, Webb M et al (2014) Psychological impact of breast asymmetry on adolescents: a prospective cohort study. Plast Reconstr Surg 134:1116-1123. https://doi.org/10.1097/PRS. 0000000000000736

8. Gabka CJ, Bohmert H (2006) Plastische und Rekonstruktive Chirurgie der Brust, 2. komplett aktualisierte Auflage, Georg Thieme Verlag, Stuttgart

9. Eisenmann-Klein M (2007) Mammaaugmentation. In: Balogh B, Berger A (eds) Mamma, Stamm, Genitale: mit 6 Tabellen. Springer, Berlin, pp 155-173

10. Chan W, Mathur B, Slade-Sharman D, Ramakrishnan V (2011) Developmental breast asymmetry. Breast J 17:391-398. https:// doi.org/10.1111/j.1524-4741.2011.01104.x

11. Piza-Katzer H (2005) Reduction mammaplasty in teenagers. Aesth Plast Surg 29:385-390. https://doi.org/10.1007/ s00266-004-0114-5

12. Brebant V, Prantl L (2019) Die Korrektur von kongenitalen Brustfehlbildungen durch autologe Fettransplantation. In: von Heimburg, Richter, Lemperle (eds) Ästhetische Chirurgie, 43. Ergänzungslieferung. ecomed MEDIZIN, Landsberg am Lech, p IX5a

13. Wiesing U (2009) Ethische Aspekte der ästhetischen Medizin. Hautarzt 60:409-413. https://doi.org/10.1007/s00105-009-1715-3

14. Neto M, Dasilva A, Garcia E et al (2007) Quality of life and selfesteem after breast asymmetry surgery. Aesthetic Surg J 27:616621. https://doi.org/10.1016/j.asj.2007.09.002

15. Cano SJ, Klassen A, Pusic AL (2009) The Science behind qualityof-life measurement: a primer for plastic surgeons. Plast Reconstr Surg 123:98e. https://doi.org/10.1097/PRS.0b013e31819565c1

16. Hsia HC, Thomson JG (2003) Differences in breast shape preferences between plastic surgeons and patients seeking breast augmentation. Plast Reconstr Surg 112:312-320. https://doi.org/10. 1097/01.PRS.0000066365.12348.A7

17. Gründl M, Eisenmann-Klein M, Prantl L (2009) Quantifying female bodily attractiveness by a statistical analysis of body 
measurements. Plast Reconstr Surg 123:1064-1071. https://doi. org/10.1097/PRS.0b013e318199f7a6

18. Chae MP, Rozen WM, Spychal RT, Hunter-Smith DJ (2016) Breast volumetric analysis for aesthetic planning in breast reconstruction: a literature review of techniques. Gland Surg 5:212226. https://doi.org/10.3978/j.issn.2227-684X.2015.10.03

19. Wesselius TS, Verhulst AC, Vreeken RD et al (2018) Accuracy of three software applications for breast volume calculations from three-dimensional surface images. Plast Reconstr Surg 142:858865. https://doi.org/10.1097/PRS.0000000000004728

20. O'Connell RL, Khabra K, Bamber JC et al (2018) Validation of the Vectra XT three-dimensional imaging system for measuring breast volume and symmetry following oncological reconstruction. Breast Cancer Res Treat 171:391-398. https://doi.org/10. 1007/s10549-018-4843-6

21. Koban KC, Frank K, Etzel L et al (2019) 3D Mammometric changes in the treatment of idiopathic gynecomastia. Aesth Plast Surg 43:616-624. https://doi.org/10.1007/s00266-019-01341-5

22. Koban KC, Schenck T, Metz PM et al (2016) Auf dem Weg zur objektiven Evaluation von Form, Volumen und Symmetrie in der Plastischen Chirurgie mittels intraoperativer 3D Scans. Handchir Mikrochir plast Chir 48:78-84. https://doi.org/10. 1055/s-0042-104506

23. Schenck TL, Koban KC, Giunta RE (2018) Ermittlung objektiver 3-D Daten zur korrekten Planung von Folgeeingriffen nach Brustrekonstruktion. In: Senologie - Zeitschrift für Mammadiagnostik und -therapie. Georg Thieme Verlag KG, p 124

24. Diaz JF (2017) Review of 494 consecutive breast augmentation patients: system to improve patient outcomes and satisfaction. Plast Reconstr Surg Glob Open. https://doi.org/10.1097/GOX. 0000000000001526

25. Camison L, Bykowski M, Lee WW et al (2018) Validation of the Vectra $\mathrm{H} 1$ portable three-dimensional photogrammetry system for facial imaging. Int J Oral Maxillofac Surg 47:403-410. https://doi. org/10.1016/j.ijom.2017.08.008

26. Hartmann R, Weiherer M, Schiltz D et al (2020) New aspects in digital breast assessment: further refinement of a method for automated digital anthropometry. Arch Gynecol Obstet. https:// doi.org/10.1007/s00404-020-05862-2

27 Heine N, Kerl S, Eisenmann-Klein M (2007) Fehlbildungen der Mamma. Frauenheilkunde up2date 1:507-521. https://doi.org/10. 1055/s-2007-981351

28. Wesselius TS, Vreeken RD, Verhulst AC et al (2018) New software and breast boundary landmarks to calculate breast volumes from 3D surface images. Eur J Plast Surg 41:663-670. https://doi. org/10.1007/s00238-018-1431-2

29. Eder M, Waldenfels F, v., Sichtermann M, et al (2011) Threedimensional evaluation of breast contour and volume changes following subpectoral augmentation mammaplasty over 6 months. J Plast Reconstr Aesthet Surg 64:1152-1160. https://doi.org/10. 1016/j.bjps.2011.03.037

30. Pusic A, Klassen A, Scott A et al (2009) Development of a new patient-reported outcome measure for breast surgery: the BREAST-Q. Plast Reconstr Surg 124:345-353. https://doi.org/ 10.1097/PRS.0b013e3181aee807
31 Pusic AL, Klassen AF, Cano SJ (2012) Use of the BREAST-Q in clinical outcomes research. Plast Reconstr Surg 129:166e-167e. https://doi.org/10.1097/PRS.0b013e3182362e65

32. Noorizadeh H, Bari BK (2020) The effect of breast augmentation surgery on quality of life, satisfaction, and marital life in married women using BREAST-Q as a validation tool. J Fam Med Prim Care 9:711-713. https://doi.org/10.4103/jfmpc.jfmpc_1013_19

33. Deschler A, Stroumza N, Pessis R et al (2020) Primary breast augmentation with autologous fat grafting alone: evaluation of patient satisfaction using the BREAST-Q. Aesthet Surg J. https:// doi.org/10.1093/asj/sjz352

34. Patlazhan G, Shkolnaya O, Torubarov I, Gomes M (2020) Our 10 years' experience in breast asymmetry correction. Aesth Plast Surg. https://doi.org/10.1007/s00266-020-01632-2

35. Kuzbari R, Deutinger M, Todoroff BP et al (1993) Surgical treatment of developmental asymmetry of the breast long-term results. Scand J Plast Reconstr Surg Hand Surg 27:203-207. https://doi. org/10.3109/02844319309078112

36. Cohen WA, Mundy LR, Ballard TNS et al (2016) The BREAST-Q in surgical research: a review of the literature 2009-2015. J Plast Reconstr Aesthet Surg 69:149-162. https://doi.org/10.1016/j.bjps. 2015.11.013

37. Sandsmark M, Amland PF, Samdal F et al (1992) Clinical results in 87 patients treated for asymmetrical breasts: a follow-up study. Scand J Plast Reconstr Surg Hand Surg 26:321-326. https://doi. org/10.3109/02844319209015278

38. Osinga R, Bodmer E, Link B et al (2014) Langzeitresultate nach chirurgischer Behandlung von Brustasymmetrien aus Sicht der Patientinnen: Eine Analyse von 51 Fällen. Handchir Mikrochir plast Chir 46:330-335. https://doi.org/10.1055/s-0034-1394426

39. Radoschewski M (2000) Gesundheitsbezogene Lebensqualität - Konzepte und Maße. Bundesgesundheitsblatt - Gesundheitsforschung - Gesundheitsschutz 43:165-189. https://doi.org/10. $1007 / \mathrm{s} 001030050033$

40. Mousavi S (2010) The ethics of aesthetic surgery. J Cutan Aesthet Surg 3:38-40. https://doi.org/10.4103/0974-2077.63396

41. Koban KC, Schenck TL, Etzel L, Giunta RE (2018) Intraoperative 3-D Symmetrieanalyse in der Brustchirurgie. In: Senologie - Zeitschrift für Mammadiagnostik und -therapie. Georg Thieme Verlag KG, p 71

42. Ji K, Luan J, Liu C et al (2014) A prospective study of breast dynamic morphological changes after dual-plane augmentation mammaplasty with 3D scanning technique. PLoS ONE. https:// doi.org/10.1371/journal.pone.0093010

43. Pietruski P, Paskal W, Paskal AM et al (2019) Analysis of the visual perception of female breast aesthetics and symmetry: an eye-tracking study. Plast Reconstr Surg 144:1257-1266. https:// doi.org/10.1097/PRS.0000000000006292

Publisher's Note Springer Nature remains neutral with regard to jurisdictional claims in published maps and institutional affiliations. 